\title{
Estimating Sway Angle of Pendulum System Using Hybrid State Observer Incorporating Continuous and Discrete Sensing Signals
}

\author{
Shuta Ano* \\ Non-member, \\ Kenta Seki ${ }^{* a)}$ \\ Senior Member \\ Michael Ruderman ${ }^{* *}$ Non-member, \\ Makoto Iwasaki* \\ Senior Member
}

(Manuscript received May 8, 2020, revised Aug. 19, 2020)

J-STAGE Advance published date : Oct. 9, 2020

\begin{abstract}
This paper presents the design of a hybrid state observer that estimates the sway angle in trolley systems with a pendulum, such as overhead cranes. In the system, sway angle signals detected by angular sensors are generally used for designing the anti-sway control of the pendulum or observing the pendulum state. By contrast, in this study, a linear state observer without sensors is applied to estimate the sway angle of the pendulum. The use of a standard asymptotic state observer leads to estimation error due to the system's nonlinearities and parametric errors. This paper proposes using a hybrid state observer design that combines discrete event sensing with a linear state observer. In the hybrid state observer, the estimation performance is improved by correcting the state of the system based on the discrete sway angle and angular velocity using discrete sensing. In addition, the parametric error of the pendulum length of the system is identified using the same hybrid setting. The effectiveness of the hybrid state observer and the parametric adaptation of the pendulum length are verified by conducting experiments using a downscaled prototype of a trolley system with a pendulum.
\end{abstract}

Keywords: pendulum system, sway angle estimation, hybrid state observer, discrete sensing signal

\section{Introduction}

Hybrid systems are systems that mix continuous and discrete dynamics ${ }^{(1)}$. Continuous dynamics correspond to phenomena expressed in equations of motion or ordinary differential equations of dynamical systems in general control theories. Discrete dynamics, on the other hand, correspond to mode transitions depending on discrete sensing or system states. Sensors typically used for discrete sensing are binaryvalued or on-off sensors based on detection principles by photoelectric, high-frequency oscillation, magnetic, and chemical. They are usually less expensive and easier to handle than regular sensors used for continuous sensing and are therefore widely used in factory automation, automotive, and chemical process systems. However, sensing by using discrete sensors only occurs when a state quantity of the object to be measured has exceeded a specific level; owing to this aspect, discrete sensors cannot measure state quantities continuously. Hence, theoretical studies have been conducted on approaches using the sensors that output trigger or binary signals, e.g., system identification ${ }^{(2)}$, continuous estimation of the state quantities $^{(3)}$, and reconstruction of the state quantities ${ }^{(4)}$. As a hybrid motion control approach, impulse or event-based control has demonstrated with promising results ${ }^{(5)-(7)}$. This approach is more attractive from the aspects of motion performance improvement. However, there are few examples of their ap-

\footnotetext{
a) Correspondence to: Kenta Seki. E-mail: k-seki@nitech.ac.jp

* Department of Electrical and Mechanical Engineering, Nagoya Institute of Technology

Gokiso, Showa, Nagoya, Aichi 466-8555, Japan

** Department of Engineering Sciences, University of Agder p.b. 422, Kristiansand, 4604 Norway
}

plication in the industry and further discussions are required.

Conveyor systems with pendulum systems, as typified by container cranes and overhead cranes, play an important role in cargo-conveying tasks in the industrial fields, including harbors and factories. Technically accomplished crane operators are needed to operate this type of machinery fast and accurately. However, in recent years, problems such as labor shortages have occurred owing to a decrease in the working population and the retirement of skilled operators; these issues are expected to further increase in the future. As a result, there is an increasing need for automated and unmanned cranes. Since the oscillation of a payload in conveyance by cranes leads to decreased efficiency and safety of conveying work, anti-sway control technology is an essential aspect in the development of automated cranes. Various antisway approaches have been studied, and the approaches can be roughly divided into feedforward and feedback control ${ }^{(8)}$. On the one hand, feedforward control involves a trolley trajectory design where suppresses load oscillations when arriving at its target position ${ }^{(9)-(11)}$. The efficacy has been verified in sway control for load. On the other hand, feedback control is an approach that feeds back the load sway angle measured using sway angle sensors ${ }^{(12)(13)}$. The control systems based on directly sensing are appreciated because they are robust against modeling errors and disturbances. Although the sensors such as potentiometers, optical/magnetic encoders, and visual cameras ${ }^{(14)}$ are used to detect the sway angle of the pendulum with load, issues remain around the space needed to install sensors, measurement conditions, cost, and sensor maintenance. A state observer is a common practice of estimating the sway angle to use as anti-sway control or monitoring the load. However, the issues with common linear 
state observers are the generation of estimation errors due to modelling/parametric errors or the system nonlinearities. To improve the estimation accuracy, a load-mounted inclinometer and observer was used in Ref. (15). In Ref. (16), a vision sensor was used to estimate the sway angle, where the long sampling time of vision sensor was compensated by time correction observer. A load position observer was implemented as Extended Kalman Filters, where the input signals are the boom tip position which can be measured using incremental encoders and the angular rope velocity, measured by gyroscopes ${ }^{(17)}$. These sensor fusion techniques combined with much cheaper and/or durable sensors appears promising for the accurate state estimation and extension of standard motion control approaches.

This paper presents an approach of a hybrid state observer design that combines discrete sensing detected by photoelectric sensors with a linear state observer for a prototype of trolley systems with pendulum systems. The objective is to accurately estimate the sway angle of the pendulum with load without using angle sensors. The sway angle of the pendulum can be continuously estimated using input and output signals of the trolley through the linear state observer. However, the modeling and parametric errors deteriorate the estimation performance. The photoelectric sensors generating the discrete sensing signals are installed on the outside of the trolley system with pendulum and detection signals are only generated when the load passes in front of the photoelectric sensors. Sway angle and angular velocity can be calculated based on these signals. The initial values of the state observer are updated to correct the sway angle estimation error based on these discrete sensing signals. This system is characterized by the fact that load detection by the photoelectric sensors is treated as a trigger. In addition, the adaptive identification of the pendulum length parameter is designed using discrete sensing signals. The usefulness of the designed hybrid state observer is verified through a sway angle estimation experiments using the prototype.

\section{Configuration and Modeling of the Prototype System}

\subsection{Prototype Configuration The external appear-} ance of the prototype system described in this paper is illustrated in Fig. 1, while the configuration of control system is shown in Fig. 2. The basic design concept can be found in Ref. (18). It consists of a combination of a linear motordriven table and a pendulum system, with the table representing the traveling trolley and the pendulum system representing the conveying load. The motor thrust reference, which is the control input calculated by the digital signal processor (DSP), is output to the servo amplifier through a DA converter. The motor driving current (motor driving thrust) is controlled with the servo amplifier through the current control system. The table is driven by motor-driven thrust and moves in the $x$ axis direction on two linear guides. The table position $x$ is measured by a linear encoder with resolution of $0.5 \mu \mathrm{m}$ and a position control system is configured based on the position signal.

The pendulum system is connected to the table through a support column and the load sways around the axis formed by the bearing installed on the support column. The sway

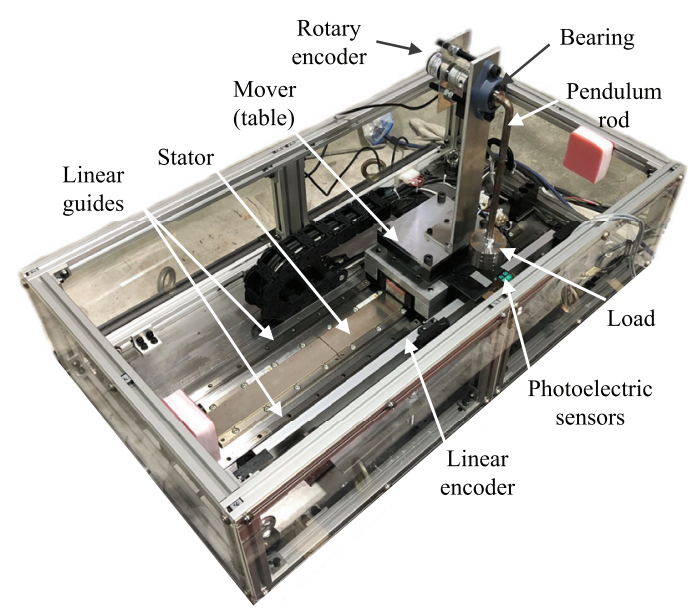

(a) Photo of prototype

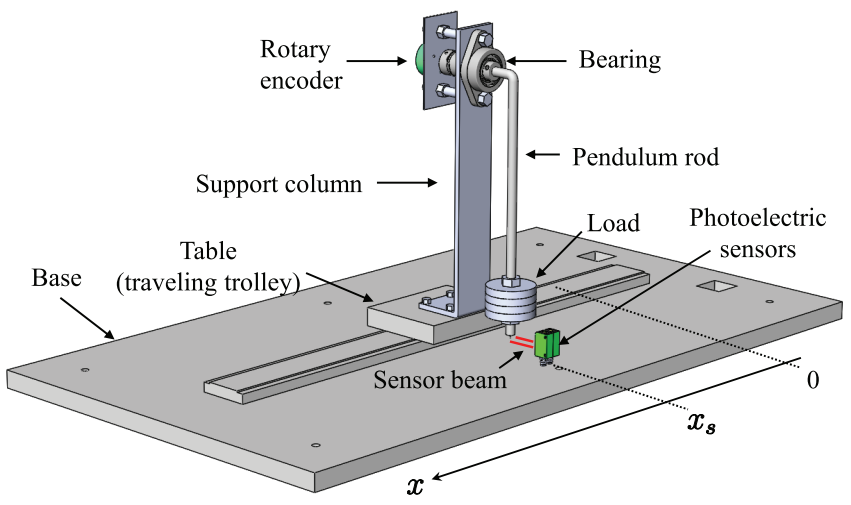

(b) Schematic view

Fig. 1. Downscaled prototype of trolley system with pendulum

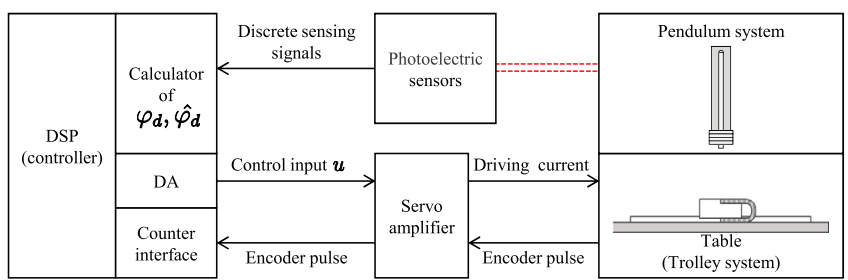

Fig. 2. Control system of trolley system with pendulum

angle of the pendulum is measured by a rotary encoder with 2400 pulse/revolution. The encoder is used for performance evaluation only and is not a regular part of the trolley system with pendulum.

Two reflective-type photoelectric sensors are installed on the external trolley base at position $x_{s}$, where a distance between the two sensors is $d_{s}=10 \mathrm{~mm}$. The response time of the sensor is $0.3 \mathrm{~ms}$. The photoelectric sensor to be used generates the output signal by detecting the change in light quantity due to the object crossing the optical axis. When sensor beam from the emitter strikes the tip of the pendulum illustrated in Fig. 1(b), the sensor outputs the signal from low-level to high-level.

2.2 Detection of Sway Angle and Angular Velocity Using Photoelectric Sensors In this paper, the sway angle and angular velocity of the pendulum is discretely detected through the detection of the tip of the pendulum by the photoelectric sensors. Using trolley position $x\left(t_{i}\right)$, position of photoelectric sensors $x_{s}$ and pendulum rod length $l$, sway angle 


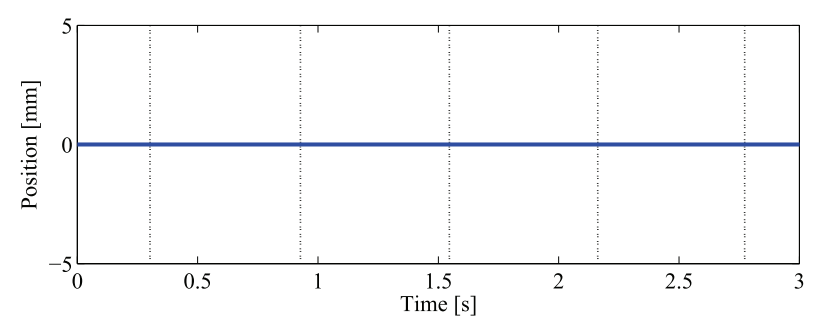

(a) Trolley position response

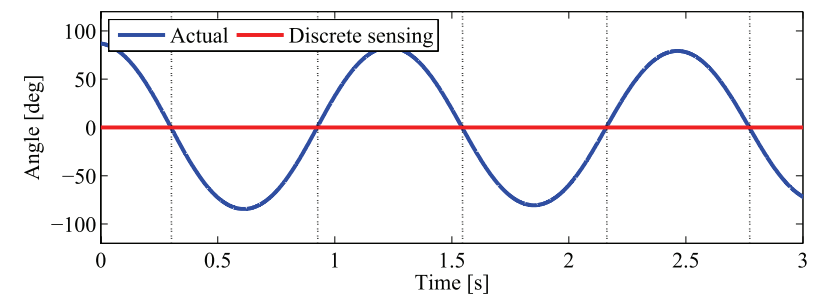

(b) Detected sway angle

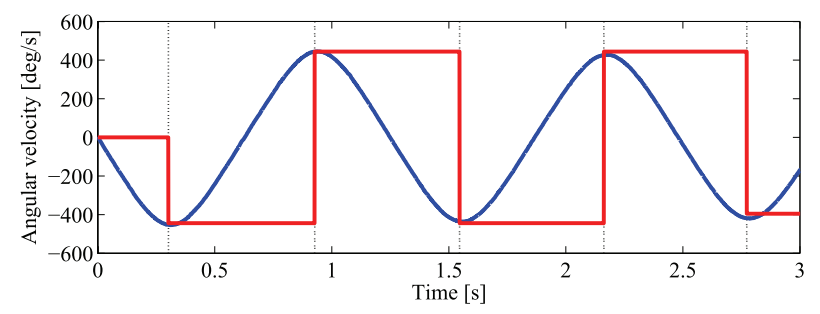

(c) Detected angular velocity

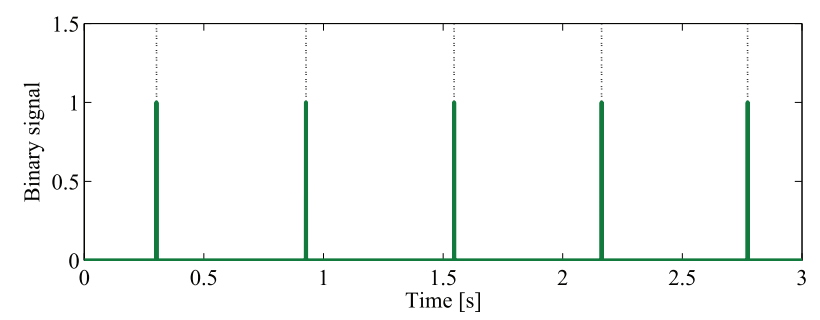

(d) Photoelectric sensors output

Fig. 3. Sway detected by photoelectric sensors for free oscillation

$\varphi_{d}\left(t_{i}\right)$ of the pendulum is obtained as follows:

$$
\varphi_{d}\left(t_{i}\right)=\sin ^{-1} \frac{x_{s}-x\left(t_{i}\right)}{l}, \cdots
$$

where $t_{i}$ is discrete sensing time by the photoelectric sensors. The time $t_{i}$ is defined as the later time out of the two sensing times generated by the pendulum passing in front of the photoelectric sensors. Tangential velocity $v_{t}\left(t_{i}\right)$ of the pendulum can be obtained with the following equation, using the distance of two photoelectric sensors $d_{s}$ and sensing time difference $\Delta t_{i}$ :

$$
v_{t}\left(t_{i}\right)=\frac{d_{s}}{\Delta t_{i}}
$$

Using the tangential velocity $v_{t}\left(t_{i}\right)$ above and pendulum rod length $l$, angular velocity $\dot{\varphi}_{d}\left(t_{i}\right)$ can be obtained by:

$$
\dot{\varphi_{d}}\left(t_{i}\right)=\frac{v_{t}\left(t_{i}\right)}{l} .
$$

Figures 3 and 4 show the computation results, using (1), (2), and (3), for sway angle and angular velocity for the free oscillation of the pendulum from 90 degrees with the trolley in a fixed state $\left(x=x_{s}\right)$, and for the sinusoidal movement of

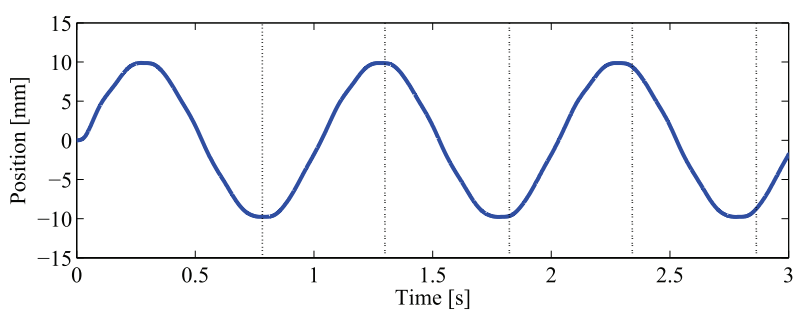

(a) Trolley position response

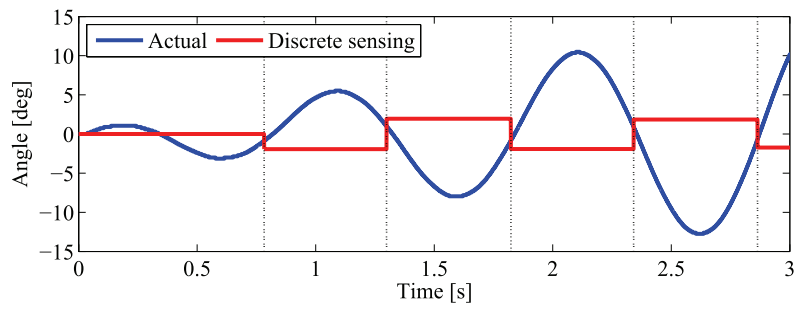

(b) Detected sway angle

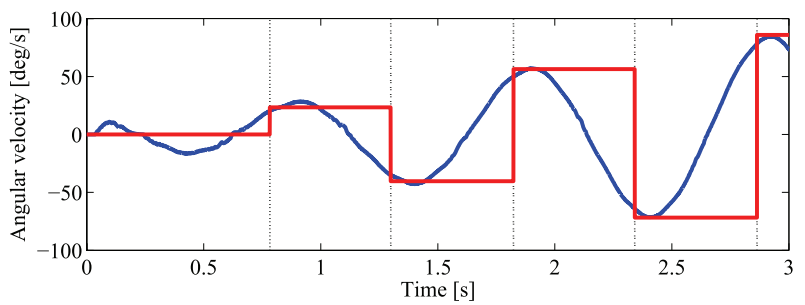

(c) Detected angular velocity

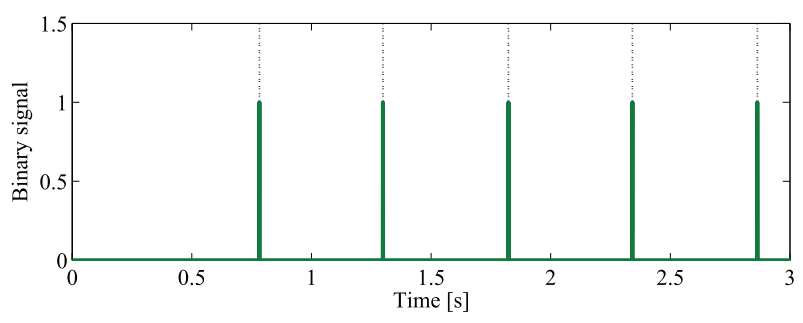

(d) Photoelectric sensors output

Fig. 4. Sway detected by photoelectric sensors for sinusoidal movement of trolley

the trolley centered around the photoelectric sensors' location. In the figures, (a), (b), (c), and (d) show the trolley position, the sway angle, the angular velocity, and output signal of the photoelectric sensors, respectively. In (b) and (c), the blue line indicates the rotary encoder measurements and the red line indicates the sway angle and angular velocity calculated with (1), (2), and (3). Based on Figs. 3 and 4, sway angle and angular velocity can be detected at the photoelectric sensors' sensing times. The sway angle and angular velocity of the pendulum can be measured continuously by the rotary encoder, but with the detection method using the photoelectric sensors, detection is only possible when the pendulum passes in front of the photoelectric sensors.

2.3 Modeling of the Prototype System The prototype system is considered as an equivalent crane system model outlined in Fig. 5.

The equation of motion is expressed as follows ${ }^{(18)}$ :

$$
\begin{aligned}
& \left(M_{C}+M_{L}\right) \ddot{x}+M_{L} l\left(\ddot{\varphi} \cos (\varphi)-\dot{\varphi}^{2} \sin (\varphi)\right)+c \operatorname{sgn}(\dot{x}) \\
& +d \dot{x}=f_{\text {in }}, \\
& M_{L} l \cos (\varphi) \ddot{x}+M_{L} l^{2} \ddot{\varphi}+M_{L} g l \sin (\varphi)+a \operatorname{sgn}(\dot{\varphi}) \\
& +b \dot{\varphi}=0 \text {, }
\end{aligned}
$$




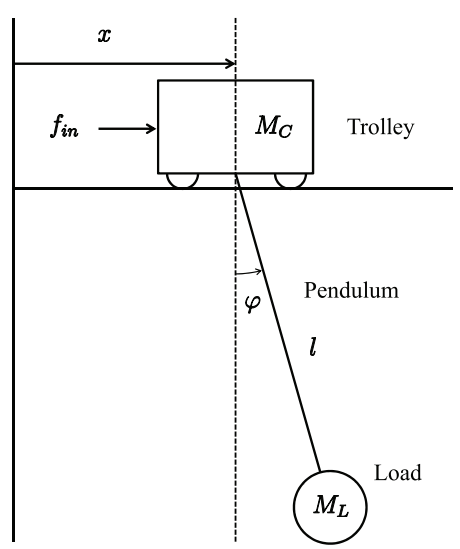

Fig. 5. Schematic view of pendulum system

Table 1. Parameters of target pendulum system

\begin{tabular}{c|c||c|c}
\hline$M_{C}[\mathrm{~kg}]$ & 14.3 & $M_{L}[\mathrm{~kg}]$ & 1.0 \\
\hline$l[\mathrm{~m}]$ & 0.289 & $a[\mathrm{Nm}]$ & $7.1 \times 10^{-3}$ \\
\hline$b[\mathrm{Nms} / \mathrm{rad}]$ & $12.2 \times 10^{-3}$ & $c[\mathrm{~N}]$ & 15.1 \\
\hline$d[\mathrm{Ns} / \mathrm{m}]$ & 16.1 & & \\
\hline
\end{tabular}

where $M_{C}$ is the trolley mass, $M_{L}$ is the load mass, $l$ is the rod length, $g$ is the gravity constant, $f_{\text {in }}$ is the trolley driving force, $c$ is the Coulomb friction coefficient for the trolley, $d$ is the viscous friction coefficient for the trolley, $a$ is the Coulomb friction coefficient around the bearing, and $b$ is the viscous friction coefficient around the bearing. The parameters are listed in Table 1. Assuming that the sway angle of the pendulum is sufficiently small $(\varphi \approx 0),(4)$ and $(5)$ can be linearized as follows:

$$
\begin{aligned}
& \left(M_{C}+M_{L}\right) \ddot{x}+M_{L} l \ddot{\varphi}+d \dot{x}=f_{i n}, \\
& M_{L} l \ddot{x}+M_{L} l^{2} \ddot{\varphi}+M_{L} g l \varphi+b \dot{\varphi}=0 .
\end{aligned}
$$

Based on the equations above, the state and output equations can be expressed as follows:

$$
\begin{aligned}
& z=\left[\begin{array}{llll}
x & \varphi & \dot{x} & \dot{\varphi}
\end{array}\right]^{T}, \\
& y=x . \cdots \cdots \cdots \cdots \cdots
\end{aligned}
$$

Here, state variable $z$ and output variable $y$ are given as follows:

$$
\begin{aligned}
& \dot{z}=A z+B f_{\text {in }} \\
& y=C z, \cdots
\end{aligned}
$$

where

$$
\begin{aligned}
A & =\left[\begin{array}{cccc}
0 & 0 & 1 & 0 \\
0 & 0 & 0 & 1 \\
0 & \frac{M_{L}}{M_{C}} g & -\frac{d}{M_{C}} & \frac{b}{M_{C} l} \\
0 & -\frac{M_{C}+M_{L}}{M_{C} l} g & \frac{d}{M_{C} l} & -\frac{b\left(M_{C}+M_{L}\right)}{M_{C} M_{L} l^{2}}
\end{array}\right], \\
B & =\left[\begin{array}{llll}
0 & 0 & \frac{1}{M_{C}} & -\frac{1}{M_{C} l}
\end{array}\right]^{T}, \\
C & =\left[\begin{array}{llll}
1 & 0 & 0 & 0
\end{array}\right] .
\end{aligned}
$$

The system expressed in (10) and (11) is observable.
2.4 Linear State Observer Design

For the system expressed in (10) and (11), the linear state observer is designed as follows ${ }^{(19)}$ :

$$
\hat{\dot{z}}=A \hat{z}+B f_{\text {in }}+L(y-C \hat{z})
$$

where $\hat{z}$ is the estimated state variable and $L$ is the observer gain. By using the state observer, sway angle $\hat{\varphi}$ can be estimated from the trolley driving force $f_{\text {in }}$ and the trolley position $x$ without using sway angle information.

\section{Hybrid State Observer Design}

Since sway angle $\varphi$ is estimated from the trolley driving force $f_{\text {in }}$ and the trolley position $x$ with the linear state observer, estimation errors occur if there are modeling errors in the observer model. In this paper, therefore, the photoelectric sensors are installed at the trolley target position and the hybrid state observer is designed to reduce estimation errors by combining their discrete sensing signals with the linear state observer. The photoelectric sensors are widely used as a detection device for positioning at a target position in the real applications ${ }^{(20)(21)}$. Sway angle and angular velocity that are computed by the discrete sensing signals are generated by the event of the pendulum passing in front of the photoelectric sensors. The initial value of the linear state observer is updated based on the discrete sensing signals to modify the estimation errors at the time of the event. If the time of discrete sensing by the photoelectric sensors is $t_{i}$, then each state variable of the pendulum system at $t<t_{i}$ can be, based on the state observer expressed in (12), computed explicitly with the following equation:

$$
\begin{aligned}
\hat{z}= & e^{(A-L C) t} z(0) \\
& +\int_{0}^{t} e^{(A-L C)(t-\tau)}\left(B f_{i n}+L y\right) d \tau
\end{aligned}
$$

The initial value in (13) is updated as in the following equation, using the discrete sensing signal at discrete sensing time $t_{i}$ :

$$
\begin{aligned}
\hat{z}= & e^{(A-L C)\left(t-t_{i}\right)} z\left(t_{i}\right) \\
& +\int_{t_{i}}^{t} e^{(A-L C)(t-\tau)}\left(B f_{i n}+L y\right) d \tau, \\
z\left(t_{i}\right)= & {\left[\begin{array}{llll}
\hat{x}\left(t_{i}\right) & \varphi_{d}\left(t_{i}\right) & \hat{x}\left(t_{i}\right) & \dot{\varphi}_{d}\left(t_{i}\right)
\end{array}\right], }
\end{aligned}
$$

where $\varphi_{d}\left(t_{i}\right)$ and $\dot{\varphi}_{d}\left(t_{i}\right)$ are the sway angle and angular velocity, respectively, detected by the photoelectric sensors. The composition of the hybrid state observer is illustrated in Fig. 6. Based on the above, the hybrid state observer calculates estimated state variable $\hat{z}$ using the trolley driving force $f_{\text {in }}$ and trolley position $x$, which are continuous sensing signals, and updates the initial values of the linear state observer using the discrete sensing signals by the photoelectric sensors.

\section{Adaptive Identification System of Pendulum Length}

Estimation performance of the linear state observer deteriorates due to modeling errors. In practice, parametric errors in the length $l$ are entirely unavoidable due to changes in the weight of the transportation object, errors in the detection position, and aged deterioration of the system with the 


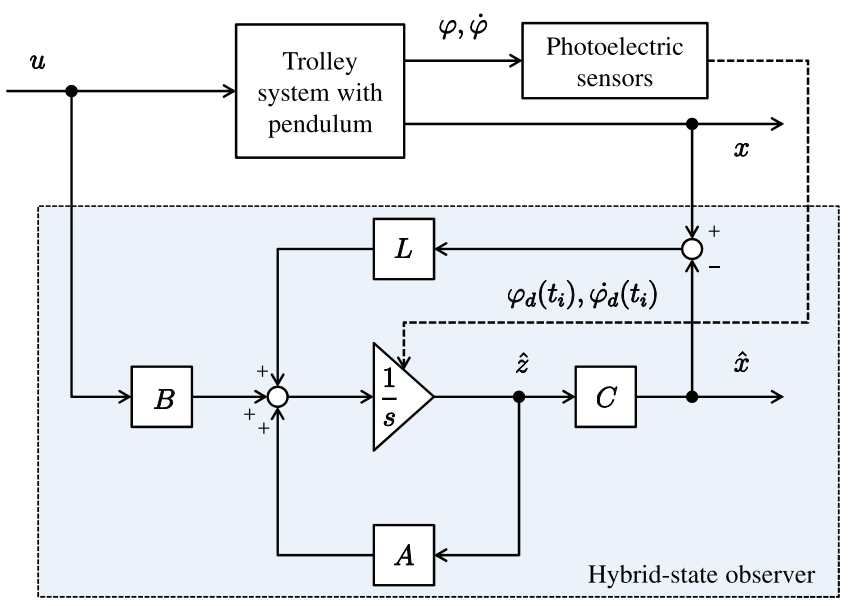

Fig. 6. Block diagram of the system with hybrid state observer

pendulum. The estimation using linear state observer, in particular, is sensitive to change in the pendulum length because it causes the natural frequency variation. As a result, estimation errors appear in the interval next to the discrete sensing time $t_{i+1}$ even if the initial value of the hybrid state observer is updated through the discrete sensing signals. In this paper, adaptive identification system is designed to identify the pendulum length using discrete sensing signals. The system is designed by focusing on the behaviors of estimated sway angle and angular velocity of the hybrid state observer which has pendulum length errors. Figures 7 and 8 show the simulation results for estimated sway angle and angular velocity of the hybrid state observer for pendulum length error of $\pm 20 \%$. The gray lines indicate the actual response detected by the rotary encoder; the blue lines indicate the hybrid state observer estimation; the red lines indicate sway angle $\varphi_{d}$ and angular velocity $\dot{\varphi}_{d}$ detected by the photoelectric sensors; and the green lines indicate the discrete sensing time by the photoelectric sensor. Figures 7 and 8 show that errors appear in the sway cycles of estimated $\hat{\varphi}$ and $\hat{\dot{\varphi}}$ due to the effect of pendulum length error between discrete sensing times $\left[t_{i}, t_{i+1}\right]$. In the case of a positive error in the observer model pendulum length for the actual pendulum length, it can be confirmed that sway angle error $e\left(t_{i}\right)=\varphi_{d}\left(t_{i}\right)-\hat{\varphi}\left(t_{i}\right)$ and angular velocity $\dot{\varphi}_{d}$ are $\left[e<0\right.$ and $\left.\dot{\varphi}_{d}<0\right]$ and $\left[e>0\right.$ and $\left.\dot{\varphi}_{d}>0\right]$. If the error is negative, it can be confirmed that they are $[e<0$ and $\left.\dot{\varphi}_{d}>0\right]$ and $\left[e>0\right.$ and $\left.\dot{\varphi}_{d}<0\right]$. In other words, whether the pendulum length error in the observer model is positive or negative, it can be identified from the relationship between sway angle error $e\left(t_{i}\right)$ and angular velocity $\dot{\varphi}_{d}$ at discrete sensing time $t_{i}$. The relationship between sway angle error $e\left(t_{i}\right)$ and angular velocity $\dot{\varphi}$ is listed in Table 2 . In Table $2, l$ indicates actual pendulum length and $\hat{l}$ indicates the pendulum length in the observer model. In this paper, the following adaptive identification equation is used to update pendulum length $\hat{l}$.

$$
\hat{l}(i+1)=\hat{l}(i)-\frac{\alpha}{i} \operatorname{sgn}\left(\dot{\varphi}_{d}\left(t_{i}\right)\right) e\left(t_{i}\right),
$$

where $\alpha$ is adaptive gain and $i(=1,2, \ldots)$ is discrete sensing number. In (16), the second term on the right decreases based on $\alpha / i$ through the increase of discrete sensing number $i$. The pendulum length $\hat{l}$ estimated by the aforementioned adaptive
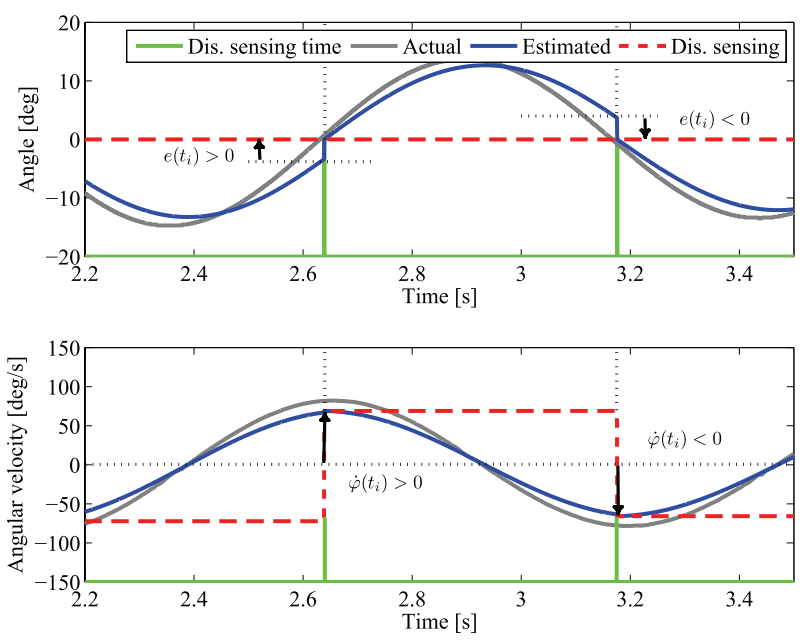

Fig. 7. Estimated sway angle and angular velocity by hybrid state observer (pendulum length $+20 \%$ )
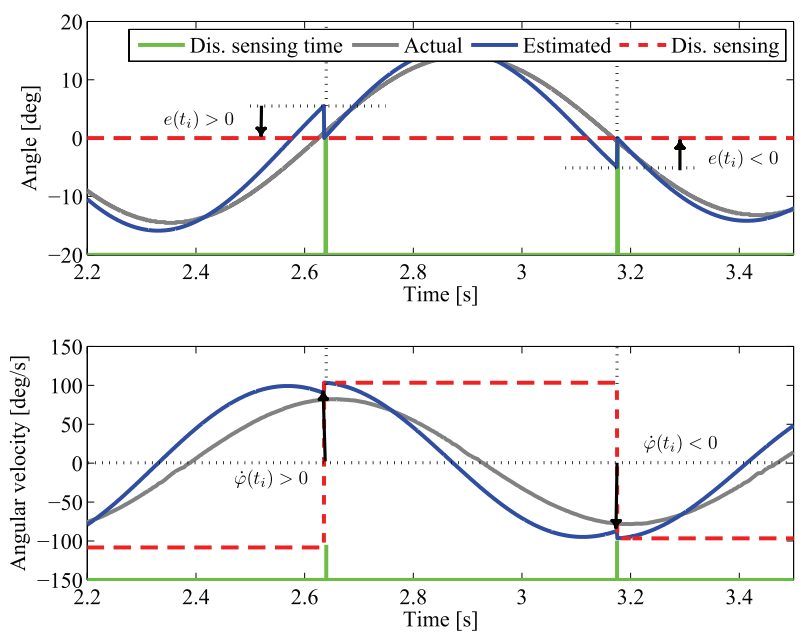

Fig. 8. Estimated sway angle and angular velocity by hybrid state observer (pendulum length $-20 \%$ )

Table 2. Relationship between sway angle error and angular velocity

\begin{tabular}{c|c|c|c|c}
\hline \hline Angular velocity & $\dot{\varphi_{d}}>0$ & $\dot{\varphi}_{d}<0$ & $\dot{\varphi}_{d}>0$ & $\dot{\varphi_{d}}<0$ \\
\hline Angle error & $e<0$ & $e>0$ & $e>0$ & $e<0$ \\
\hline \hline Rod length & \multicolumn{2}{|c|}{$\hat{\imath}>l$} & \multicolumn{2}{c}{$\hat{\imath}<l$} \\
\hline
\end{tabular}

identification system is used to update the linear state observer model in (12). In practice, the discrete sensing number $i$ continues to increase during operation. Therefore, $i$ should be reset when the next or a new operation is started.

\section{Experimental Verification}

The prototype system in Fig. 1 is used to verify the proposed hybrid state observer and the pendulum length adaptive system. As illustrated in Fig. 9, in the experiments, sway angle is estimated when the trolley is driven through an Sshape reference at target position $100 \mathrm{~mm}$ as shown in gray line of Fig. 10(a) and when the trolley is driven through a sinusoidal reference with amplitude $10 \mathrm{~mm}$ and frequency $1 \mathrm{~Hz}$ as shown in gray line of Fig. 10(b). The position control system of the trolley is designed to achieve the precise tracking performance for the position reference (see Ref. (22) for 


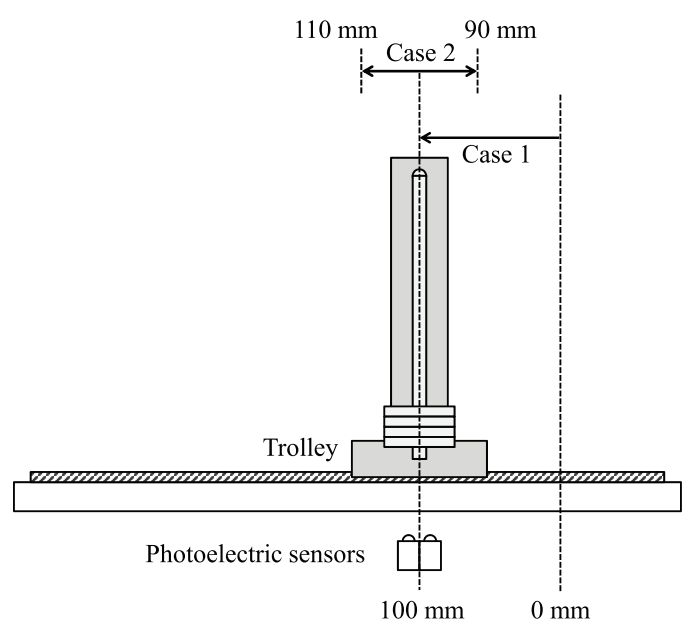

Fig. 9. Trolley motion in experiments

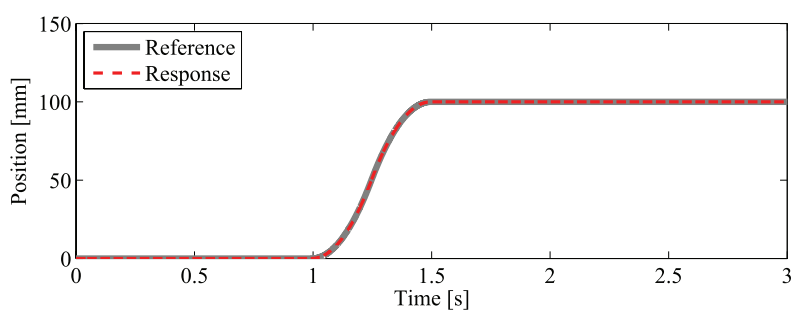

(a) Case 1

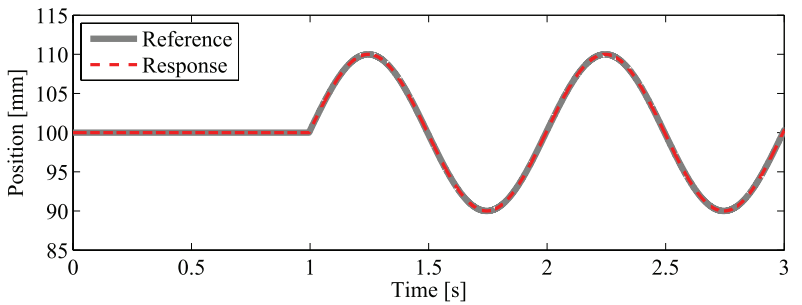

(b) Case 2

Fig. 10. References and responses of the trolley

details). Broken red lines of Fig. 10 indicate the position responses of the trolley.

To verify the robustness against pendulum length error, conventional linear state observer and proposed hybrid state observer to be compared are designed based on the model with pendulum length error of $+20 \%$. The observer gain $L$ is determined by considering the pole assignment of -10 for all poles. The adaptive gain is set as 0.8. Experimental results are shown in Figs. 11 and 12. The figures labeled (a) show estimated sway angle and the ones labeled (b) show adaptive identification of pendulum length. The black lines in (a) indicate the sway angle measured by the rotary encoder; the blue line indicates the estimation result using conventional linear state observer; the red line indicates the estimation result using the proposed hybrid state observer; and the green line indicates the discrete sensing time. Figures 11 and 12 shows that the sway angle estimation error by the hybrid state observer is smaller than with the conventional linear state observer. Moreover, it can be confirmed that with the proposed pendulum length adaptive identification system, the parameter of the length is asymptotic to the actual one. Thus, the usefulness of the proposed approach for sway angle estimation has been verified.
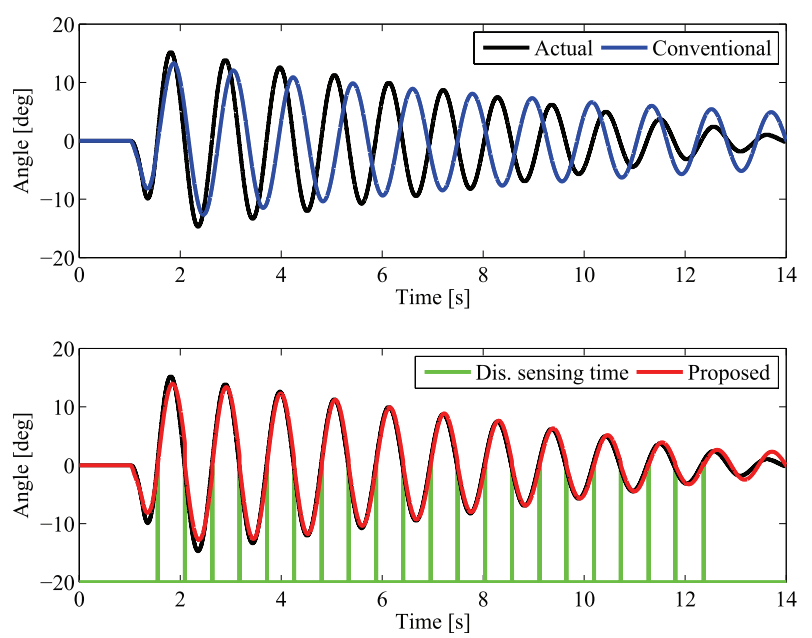

(a) Estimated sway angle

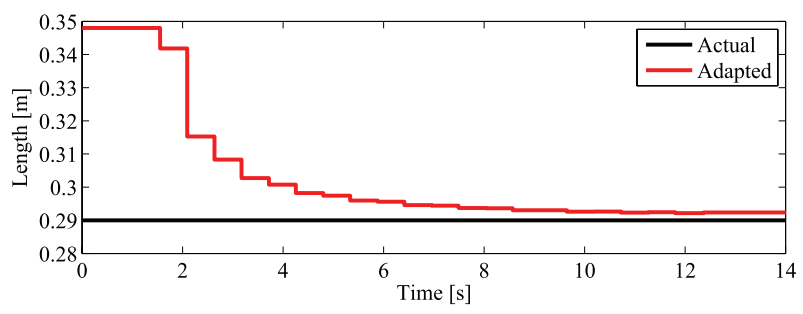

(b) Parameter identification of pendulum length

Fig. 11. Experimental results of case 1
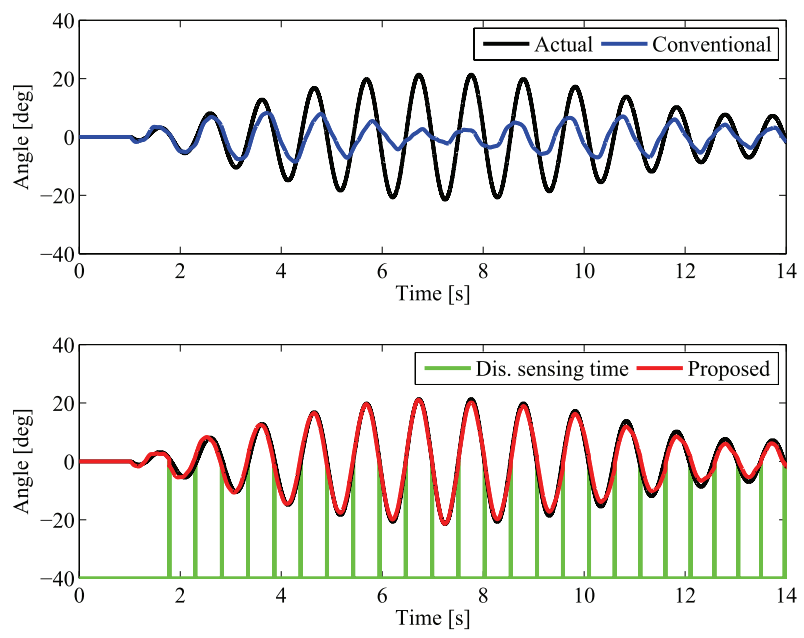

(a) Estimated sway angle

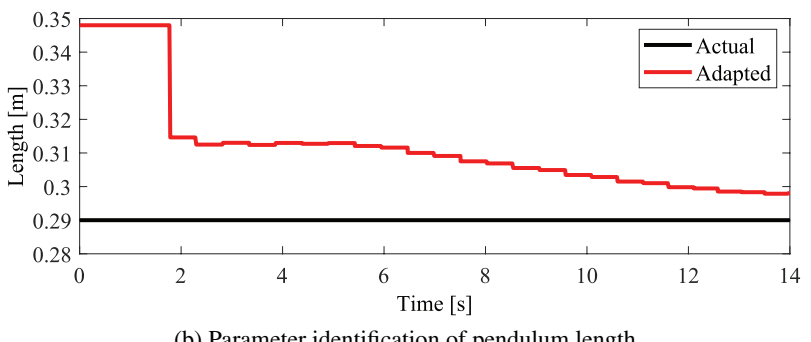

(b) Parameter identification of pendulum length

Fig. 12. Experimental results of case 2

\section{Conclusions}

In this paper, we designed a hybrid state observer incorporating continuous and discrete sensing for the estimation of the sway angle in the trolley systems with the pendulum. 
Although the sway angle of the pendulum can be estimated using input and output signals of the trolley through the linear state observer based on the model of the trolley system with pendulum, the modeling and parametric errors deteriorate the estimation performance. Two photoelectric sensors, which are discrete sensors, were placed at the trolley target location, and the sway angle and angular velocity of the pendulum were discretely computed based on the signals. To correct the sway angle estimation error, the initial value of the linear state observer was updated using the discrete sensing signals at the sensing time. Moreover, we designed a system for the adaptive identification of the pendulum length parameter using discrete sensing signals. The usefulness of proposed system was verified by conducting the experiments using the prototype based on the trolley system with pendulum.

\section{References}

(1) A.J. van der Schaft and J.M. Schumacher: "An Introduction to Hybrid Dynamical Systems", Springer-Verlag (2000)

( 2 ) L.Y. Wang, J.F. Zhang, and G.G. Yin: "System Identification Using Binary Sensors", IEEE Trans. on Automatic Control, Vol.48, No.11, pp.1892-1907 (2003)

( 3 ) X.D. Koutsoukos: "Estimation of hybrid systems using discrete sensors", IEEE 42nd Conference on Decision and Control, pp.155-160 (2003)

( 4 ) G. Xu, L.Y. Wang, and G. Yin: "State reconstruction for linear time-invariant systems with binary-valued output observations", IEEE 46th Conference on Decision and Control, pp.4305-4310 (2007)

( 5 ) J. Lunze and D. Lehmann: "A state-feedback approach to event-based control", Automatica, Vol.46, No.1, pp.211-215 (2010)

( 6 ) N. van de Wouw and R.I. Leine: "Robust impulsive control of motion systems with uncertain friction", International Journal of Robust and Nonlinear Control, Vo.22, No.4, pp.369-397 (2012)

( 7 ) M. Ruderman: "Impulse-based hybrid motion control", IEEE 43rd Annual Conference of the Industrial Electronics Society, pp.4049-4054 (2017)

( 8 ) E.M. Abdel-Rahman, A.H. Nayfeh, and Z. Masoud: "Dynamics and Control of Cranes: A Review", Journal of Vibration and Control, Vol.9, No.7, pp.863-908 (2003)

(9) W. Singhose, L. Porter, M. Kenison, and E. Kriikku: "Effects of hoisting on the input shaping control of gantry cranes", Control Engineering Practice, Vol.8, No.10, pp.1159-1165 (2000)

(10) H.H. Lee: "A New Motion-Planning Scheme for Overhead Cranes With High-Speed Hoisting", ASME Journal of Dynamics, Systems, Measurement, and Control, Vol.126, No.2, pp.359-364 (2004)

(11) A. Piazzi and A. Visioli: "Optimal dynamic-inversion-based control of an overhead crane", IEE Proceedings-Control Theory and Applications, Vol.149, No.5, pp.405-411 (2002)

(12) W. Chen and M. Saif: "Output Feedback Controller Design for a Class of MIMO Nonlinear Systems Using High-Order Sliding-Mode Differentiators With Application to a Laboratory 3-D Crane", IEEE Trans. on Industrial Electronics, Vol.55, No.11, pp.3985-3997 (2008)

(13) N. Sun, Y. Fang, and H. Chen: "A New Antiswing Control Method for Underactuated Cranes With Unmodeled Uncertainties Theoretical Design and Hardware Experiments", IEEE Trans. on Industrial Electronics, Vol.62, No.1, pp.453-465 (2015)

(14) Y. Yoshida and K. Tsuzuki: "Visual Tracking and Control of a Moving Overhead Crane Load", IEEE 9th International Workshop on Advanced Motion Control, pp.630-635 (2006)

(15) Y.S. Kim, K.S. Hong, and S.K. Sul: "Anti-Sway Control of Container Cranes: Inclinometer, Observer, and State Feedback", Int. Journal of Control, Automation, and Systems, Vol.2, No.4, pp.435-449 (2004)

(16) H. Sano, K. Ohishi, T. Kaneko, and H. Mine: "Anti Sway Crane Control Based on Dual State Observer with Sensor-Delay Correction", IEEE 11th International Workshop on Advanced Motion Control, pp.679-684 (2010)

(17) U. Schaper, C. Sagert, O. Sawodny, and K. Schneider: "A load position observer for cranes with gyroscope measurements", Proc. of the 18th IFAC World Congress, pp.3563-3568 (2011)

(18) W. Karimi, M. Ruderman, K. Seki, and M. Iwasaki: "Experimental framework of traveling trolley with swinging load for hybrid motion control", IEEE 15th International Workshop on Advanced Motion Control, pp.60-65 (2018)
(19) D.G. Luenberger: "An Introduction to Observers", IEEE Transactions on Automatic Control, Vol.16, No.6, pp.596-602 (1971)

(20) T. Murosaki, et al.: "Sensor-less Compact Assembly Machine with CCD", Proc. of International Conference on Leading Edge Manufacturing in 21st century, pp.475-478 (2005)

(21) H. Nogami: "Stable reading for UHF RFID", OMRON TECHNICS, Vol.51, pp.1-7 (2019)

(22) K. Seki, Y. Tsuchimoto, and M. Iwasaki: "Feedforward Compensation by Specified Step Settling with Frequency Shaping of Position Reference", IEEE Trans. on Industrial Electronics, Vol.61, No.3, pp.1552-1561 (2014)

Shuta Ano (Non-member) received the B.S. and M.S. degrees in elec-

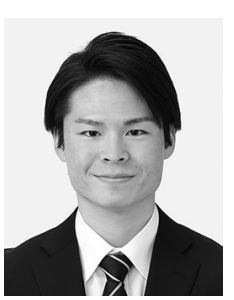
trical and mechanical engineering from Nagoya Institute of Technology, Nagoya, Japan, in 2018 and 2020 From 2020, he has been with Toyota Industries Corporation, Ltd., Japan. His research interest is design and estimation for motion control and hybrid control systems.

Kenta Seki (Senior Member) received the B.S., M.S., and Dr.Eng.

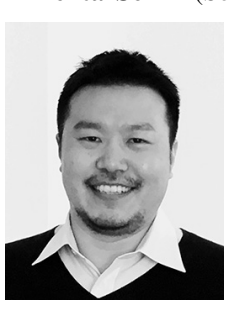
degrees in electrical and computer engineering from Nagoya Institute of Technology, Nagoya, Japan, in 2000, 2002 and 2009, respectively. From 2002 to 2006, he was with the Mechanical Engineering Research Laboratory, Hitachi, Ltd., Ibaraki, Japan. From March 2006, he joined a Research Associate with the Project Research Laboratory on Motion Systems, Nagoya Institute of Technology, Nagoya, Japan, where he is currently an Associate Professor with the Department of Electrical and Mechanical Engineering. His current research interests are the mechatronics system design and modeling and control of smart materials. Prof. Seki is a member of the Institute of Electrical Engineering of Japan, the Society of Instrument and Control Engineers, and the Japan Society of Mechanical Engineers.

Michael Ruderman (Non-member) received the Dr.-Ing. in electri-

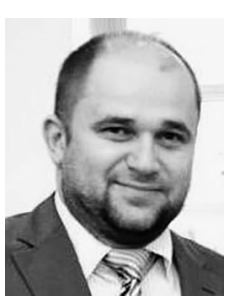
cal engineering from the Technical University (TU) Dortmund, Dortmund, Germany, in 2012. During 2006-2013, he was a Research Associate with the Institute of Control Theory and Systems Engineering, TU Dortmund. In 2013-2015, he was with Nagoya Institute of Technology, Nagoya, Japan, as specially appointed Assistant Professor. In 2015 he was specially appointed Associate Professor with the Department of Electrical Engineering, Nagaoka University of Technology, Nagaoka, Japan, before joining in the same year the University of Agder (UiA), Grimstad, Norway. Since 2020 he is a full professor at UiA, teaching control theory in Master and PhD programs. His current research interests are in the motion control, robotics, nonlinear systems with memory, and hybrid control systems.

Makoto Iwasaki (Senior Member) received the B.S., M.S., and

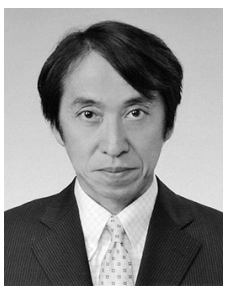
Dr.Eng. degrees in electrical and computer engineering from Nagoya Institute of Technology, Nagoya, Japan, in 1986, 1988, and 1991, respectively. Since 1991, he has been with the Department of Computer Science and Engineering, Nagoya Institute of Technology, where he is currently a Professor. His current research interests are the applications of control theories to linear/nonlinear modeling and precision positioning, through various collaborative research activities with industries. Prof. Iwasaki is a member of the Institute of Electrical Engineering of Japan and the Japan Society for Precision Engineering. 